\title{
Curvature-matter coupling effects on axial gravitational waves
}

\author{
M. Sharif ${ }^{\mathrm{a}}$, Aisha Siddiqa ${ }^{\mathrm{b}}$ \\ Department of Mathematics, University of the Punjab, Quaid-e-Azam Campus, Lahore 54590, Pakistan
}

Received: 4 June 2018 / Accepted: 28 August 2018 / Published online: 6 September 2018

(C) The Author(s) 2018

\begin{abstract}
In this paper, we investigate propagation of axial gravitational waves in the background of flat FRW universe in $f(R, T)$ theory. The field equations are obtained for unperturbed as well as axially perturbed FRW metric. These field equations are solved simultaneously to obtain the unknown perturbation parameters. We find that the assumed perturbations can affect matter as well as four velocity. Moreover, ignoring the material perturbations we explicitly obtain an expression for four velocity. It is concluded that axial gravitational waves in the curvature-matter coupling background can produce cosmological rotation or have memory effect if the wave profile has discontinuity at the wave front.
\end{abstract}

\section{Introduction}

The discovery of cosmic expansion is a big achievement as well as the most fascinating area of research. Researchers introduced different approaches to investigate the reason behind this phenomenon by modifying matter or geometric part of the Einstein-Hilbert action leading to modified matter models or modified theories of gravity, respectively. Examples of modification in geometric part are $f(R)$ [1], $f(G)$ [2] and $f(R, T)$ [3] theories of gravity where $R, G$ and $T$ denote Ricci scalar, Gauss-Bonnet invariant and trace of the energy-momentum tensor. While examples of modified matter models are quintessence [4,5], phantom [6], K-essence [7], holographic dark energy [8,9] and Chaplygin gas models [10-12].

The simplest generalization of general relativity (GR) is obtained by replacing $R$ with its generic function named as $f(R)$ in the Einstein-Hilbert action leading to $f(R)$ theory. Many astrophysical as well as cosmological aspects have been investigated within the framework of this theory [13, 14]. Harko et al. [3] proposed $f(R, T)$ gravity which is a

\footnotetext{
a e-mail: msharif.math@pu.edu.pk

b e-mail: aisha.siddiqa17@yahoo.com
}

curvature-matter coupling theory. This can produce a matter dependent deviation from geodesic motion and also help to study dark energy, dark matter interactions as well as latetime acceleration [15].

Different aspects of cosmic and stellar evolution have been studied in $f(R, T)$ gravity. Sharif and Zubair [16] investigated the validity of second law of thermodynamics for phantom as well as non-phantom phases. Shabani and Farhoudi [17] explored viability of some $f(R, T)$ gravity models by solar system constraints. Yousaf et al. [18] investigated the stability of cylindrical symmetric stellar configurations by inducing perturbations in this theory. We have studied physical characteristics of charged [19] as well as uncharged stellar structure [20] in this gravity.

The fluctuations in the fabric of spacetime produced by massive celestial objects are known as gravitational waves (GWs). The significance of GWs comes from the fact that they lead to new techniques to explore cosmic issues. The observations of GWs can help us to study the individual sources of GWs that give information about structure as well as kinematics of the cosmos. The observation of a stochastic background of GWs of cosmological origin can provide information about initial structure formation. These detections have inaugurated a new era of astronomy as well as the possibility to investigate gravity in extreme gravity regimes.

After a long history of struggles (from Weber bars to advanced laser interferometers), scientific efforts came true and GWs are finally detected by earth-based detectors. Some of the observed GWs signals by the LIGO-VIRGO collaboration are GW150914 [21], GW170104 [22] and GW170817 [23]. The origin of these signals is the merging binaries of black holes and neutron stars which release energy in the form of GWs. The most recent signal (GW170817) [23] is consistent with the binary neutron star inspiral. It has an association with gamma ray burst signal GRB170817A detected by Fermi-GBM and provides the first direct evidence of gamma ray bursts during the mergence of two neutron stars. 
The phenomenon of GWs has become a topic of central importance in cosmology nowadays. The polarization of a GW provides information for its geometrical orientation. Kausar et al. [24] explored polarization modes of GWs in $f(R)$ theory and found two modes other than GR. Alves et al. [25] evaluated these modes for $f(R, T)$ and $f\left(R, T^{\phi}\right)$ theories (here $\phi$ represents scalar field). They concluded that in vacuum the former one produces the same results as $f(R)$ while the polarization modes in $f\left(R, T^{\phi}\right)$ gravity depend upon the expression of $T^{\phi}$. We have shown that axially symmetric dust fluid with dissipation behave as a source of gravitational radiation in $f(R)$ theory [26]. We have also studied polarization modes of GWs for some viable $f(R)$ models [27].

Regge and Wheeler [28] studied the stability of Schwarzschild singularity by introducing small perturbations in the form of spherical harmonics producing odd and even waves. They found that these disturbances oscillate around equilibrium state and do not grow with time showing the stability of Schwarzschild singularity. Zerilli [29] analyzed the emission of gravitational radiation when a black hole swallows a star. He did this analysis by considering the problem of a particle falling into a Schwarzschild black hole and perturbations introduced by Regge and Wheeler as well as corrected the even wave propagation equation derived in [28]. The energy carried by GWs is the gravitational radiation. Hawking [30] investigated gravitational radiation produced by colliding black holes and Wagoner [31] discussed these radiation for accreting neutron stars.

Malec and Wylężek [32] used the wavelike perturbations proposed by Regge and Wheeler in the Schwarzschild spacetime to study the GW propagation in cosmological context. They investigated Huygens principle for cosmological GWs in Regge-Wheeler gauge and found that this principle is satisfied in radiation dominated era while it does not hold in matter dominated universe. Otakar [33] explored the GW propagations in higher dimensions using axial perturbations proposed by Regge-Wheeler. They showed that in braneworld scenario the Huygens principle seems to be satisfied for high multipoles in contrast with four dimensions. Viaggiu [34] studied propagations of axial and polar GWs proposed in [28], in de Sitter universe using the Laplace transformation. Kulczycki and Malec also [35] studied the perturbations induced by axial and polar GWs in FRW universe. They concluded that Huygens principle has the same status for both types of waves, it is valid for radiation era while it is broken elsewhere. The same authors [36] discussed cosmological rotation of radiation matter induced by axial GWs. However, axial and polar perturbations have also been studied using gauge-invariant quantities [37-40]. In [40], the authors investigated the cosmological perturbations in the context of Lemaitre-Tolman spacetime. In case of axial modes, their equations (restricted to FRW metric) coincide with that of [35].

The issues of cosmological rotation induced by GWs and validity of Huygens principle in Regge-Wheeler gauge have not yet been studied in the framework of modified theories. In the present work, we induce the axial perturbations (which change the geometry from spherical to axial) introduced by Regge and Wheeler [28] in the flat cosmological as well as curvature-matter coupling backgrounds. Since the FRW universes are conformally flat, these distortions are linked with the axial GWs. These disturbances are may be the consequence of non-gravitational forces (electromagnetic forces, nuclear forces) associated with brutal astrophysical events. The non-symmetric explosion of a supernova could be an example for the production of such type of waves. We focus on the axial wave perturbations induced in flat cosmos consisting of perfect fluid. The paper is arranged as follows. In the coming section, we discuss the background FRW cosmology in $f(R, T)$ theory. In Sect. 3, we define the perturbations in FRW metric as well as matter variables and formulate the corresponding field equations. The unknown perturbation parameters are found in Sect. 4. Finally, we summarize and conclude the results in the last section.

\section{FRW cosmology and $f(R, T)$ gravity}

In order to discuss the wave propagation in FRW universe, we consider the FRW metric in conformal coordinates $(\eta, r, \theta, \phi)$ as

$d s^{2}=a^{2}(\eta)\left(-d \eta^{2}+d r^{2}+r^{2} d \theta^{2}+r^{2} \sin ^{2} \theta d \phi^{2}\right)$,

where $\eta$ is the conformal time coordinate related to the ordinary time by the relation

$\eta=\int \frac{d t}{a}$,

such that the conformal Hubble parameter $H$ is related with the ordinary Hubble parameter $\mathcal{H}$ by

$\mathcal{H}=\frac{H}{a}$

We consider matter as perfect fluid defined by the energymomentum tensor

$T_{\mu \nu}=\left(\rho_{0}+p_{0}\right) V_{\mu} V_{\nu}+p g_{\mu \nu}$,

where $V_{\mu}, \rho_{0}$ and $p_{0}$ stand for four-velocity as well as background unperturbed density and pressure, respectively. 
The action integral for $f(R, T)$ theory is

$S=\int d^{4} x \sqrt{-g}\left[\frac{1}{16 \pi} f(R, T)+\mathcal{L}_{m}\right]$.

where $g$ is the determinant of the metric tensor and $\mathcal{L}_{m}$ is the matter Lagrangian density. The field equations for this action are

$$
\begin{aligned}
& f_{R} R_{\mu \nu}-\frac{1}{2} g_{\mu \nu} f-\left(\nabla_{\mu} \nabla_{\nu}-g_{\mu \nu} \square\right) f_{R} \\
& \quad=8 \pi T_{\mu \nu}-f_{T}\left(\Theta_{\mu \nu}+T_{\mu \nu}\right),
\end{aligned}
$$

where $f_{R}=\frac{\partial f}{\partial R}, f_{T}=\frac{\partial f}{\partial T}$ and $\Theta_{\mu \nu}=-2 T_{\mu \nu}+\mathcal{L}_{m} g_{\mu \nu}$. In this paper, we consider $f(R, T)=R+2 \lambda T$ [3] to investigate the role of curvature-matter coupling on the propagation of GWs. This model can discuss the accelerated expansion by producing a power-law like scale factor. It also has a correspondence with $\Lambda \mathrm{CDM}$ model by considering the cosmological constant as a function of trace $T$ or $\Lambda(T)$ gravity by Poplawski [41]. The choices for matter Lagrangian density $\mathcal{L}_{m}$ are $p_{0}$ or $-\rho_{0}$. However, it is shown that these two densities yield the same results for minimal curvature-matter coupling if the matter under discussion is perfect fluid [42]. So the assumption $\mathcal{L}_{m}=p_{0}$ and the model $f(R, T)=R+2 \lambda T$ simplify the field equations as

$$
G_{\mu \nu}=(8 \pi+2 \lambda) T_{\mu \nu}-2 \lambda p g_{\mu \nu}+\lambda T g_{\mu \nu} .
$$

This yields the following independent field equations for the metric (1)

$$
\begin{aligned}
3 H^{2} & =(8 \pi+3 \lambda) \rho_{0} a^{2}-\lambda p_{0} a^{2}, \\
-2 \dot{H}-H^{2} & =(8 \pi+3 \lambda) p_{0} a^{2}-\lambda \rho_{0} a^{2},
\end{aligned}
$$

here dot denote the derivative with respect to the conformal time $\eta$.

In further discussion, we consider the GWs in radiation dominated era so using the equation of state $(\operatorname{EoS}) p_{0}=\frac{\rho_{0}}{3}$, the field equations (8) and (9) give the following differential equation in $H$

$2 \dot{H}+\frac{6 \pi+\lambda}{3 \pi+\lambda} H^{2}=0$,

which yields the scale factor

$a(\eta)=c_{1} \eta^{\frac{6 \pi+2 \lambda}{6 \pi+\lambda}}$

where $c_{1}$ is constant of integration. The covariant derivative of the field equations is

$$
\begin{aligned}
\nabla^{\mu} T_{\mu \nu}= & \frac{f_{T}}{8 \pi-f_{T}}\left[\left(T_{\mu \nu}+\Theta_{\mu \nu}\right) \nabla^{\mu} \ln f_{T}\right. \\
& \left.+\nabla^{\mu} \Theta_{\mu \nu}-\frac{1}{2} g_{\mu \nu} \nabla^{\mu} T\right] .
\end{aligned}
$$

Using Eqs. (1), (4), the model $f(R, T)=R+2 \lambda T$ and $p_{0}=$ $\frac{\rho_{0}}{3}$, Eq. (11) produces the following differential equation in $\rho_{0}$

$\dot{\rho}+3 \frac{8 \pi+\lambda}{6 \pi+\lambda} H \rho_{0}=0$,

whose solution is

$\rho_{0}=c_{2} a^{\frac{-3(8 \pi+\lambda)}{6 \pi+\lambda}}$,

$c_{2}$ is again an integration constant. These values of scale factor and density are used in the further mathematics.

\section{Axial perturbations in FRW spacetime}

In this section, we first briefly discuss perturbations used to study the effects of GWs. Here, the background metric $g_{\mu \nu}$ is the FRW spacetime and $h_{\mu \nu}$ are the corresponding perturbations in the metric tensor due to GWs such that we have

$g_{\mu \nu}^{(\text {perturb })}=g_{\mu \nu}^{(\text {flat })}+e h_{\mu \nu}+O\left(e^{2}\right)$,

where $e$ is a small parameter (it measures strength of perturbations and the terms involving $O\left(e^{2}\right)$ are neglected).

We follow the Regge-Wheeler [28] perturbation scheme to investigate the wavelike fluctuations. To obtain explicit expressions for the components of $h_{\mu \nu}$ in terms of four coordinates $\left(x^{0}=\eta, x^{1}=r, x^{2}=\theta, x^{3}=\phi\right)$, they expressed them in the form of spherical harmonics. The symmetry of the metric tensor allows the angular momentum to be defined. The angular momentum is discussed by assuming the rotations on a 2D-manifold with $\eta=$ constant and $r=$ constant. The components of $h_{\mu \nu}$ have different transformations under a rotation of the frame. Among the ten independent components of the tensor $h_{\mu \nu}$, the components $h_{00}, h_{01}, h_{11}$ transform like scalars (as $x^{0}=\eta$ and $x^{1}=r$ are constants and do not change during rotation), $h_{02}, h_{03}, h_{12}, h_{13}$ change like vectors (as $x^{2}$ and $x^{3}$ are changed during rotation) while $h_{22}, h_{23}, h_{33}$ transform like tensors. Further, these scalars, vectors and tensors are expressed in terms of spherical harmonics $Y_{L}{ }^{M}$ where $L$ is the angular momentum with the projection $M$ on $z$-axis. After this, they expressed the perturbation matrix $h_{\mu \nu}$ in terms of odd and even parity waves. In this paper, we only consider the odd or axial wave perturbations defined by the matrix [28]

$h_{\mu \nu}=\partial_{\theta} Y \sin \theta\left(\begin{array}{cccc}0 & 0 & 0 & k_{0} \\ 0 & 0 & 0 & k_{1} \\ 0 & 0 & 0 & 0 \\ k_{0} & k_{1} & 0 & 0\end{array}\right)$,

with $k_{0}=k_{0}(\eta, r)$ and $k_{1}=k_{1}(\eta, r)$. Here we are considering the odd waves corresponding to $m=0$, which are 
discussed by Regge and Wheeler [28] so that $\phi$ disappears in calculations. Also, for the wavelike solution the index $l$ exceeds one, i.e., $Y=Y_{l 0} ; l=2,3, \ldots$. The resulting axially perturbed FRW spacetime in Regge-Wheeler gauge is defined by

$$
\begin{aligned}
d s^{2}= & -a^{2}(\eta) d \eta^{2}+2 e k_{0} \partial_{\theta} Y \sin \theta d \eta d \phi+a^{2}(\eta) d r^{2} \\
& +2 e k_{1} \partial_{\theta} Y \sin \theta d r d \phi \\
& +a^{2}(\eta) r^{2} d \theta^{2}+a^{2}(\eta) r^{2} \sin ^{2} \theta d \phi^{2}+O\left(e^{2}\right) .
\end{aligned}
$$

The perturbations in the material quantities are defined as follows [35]

$$
\begin{aligned}
& \rho=\rho_{0}(1+e \Delta(\eta, r) Y)+O\left(e^{2}\right), \\
& p=p_{0}(1+e \Pi(\eta, r) Y)+O\left(e^{2}\right),
\end{aligned}
$$

where $\rho_{0}$ and $p_{0}$ are the background density and pressure. The fluid may or may not be comoving in the perturbed scenario so the perturbed components of four velocity are taken as [35]

$$
\begin{aligned}
& V_{0}=\frac{2 g_{00}^{(0)}+e k_{00}}{2 a(\eta)}+O\left(e^{2}\right), \\
& V_{1}=e a(\eta) w(\eta, r) Y+O\left(e^{2}\right), \\
& V_{2}=e v(\eta, r) Y^{\prime}+O\left(e^{2}\right), \\
& V_{3}=e \sin \theta u(\eta, r) Y^{\prime}+O\left(e^{2}\right),
\end{aligned}
$$

where $V_{\alpha} V^{\alpha}=-1+O\left(e^{2}\right)$. The field equations for the perturbed metric (15) as well as corresponding perturbed matter are

$$
\begin{aligned}
3 H^{2}= & {\left[(8 \pi+3 \lambda) \rho_{0}-\lambda p_{0}+(8 \pi+3 \lambda) \rho_{0} e \Delta Y\right.} \\
& \left.-\lambda p_{0} e \Pi Y\right] a^{2}, \\
w(8 \pi & +2 \lambda)\left(\rho_{0}+p_{0}\right)=0, \\
v(8 \pi & +2 \lambda)\left(\rho_{0}+p_{0}\right)=0, \\
-2 \dot{H}-H^{2}=a^{2}\left[(8 \pi+3 \lambda) p_{0}-\lambda \rho_{0}\right. & \left.+(8 \pi+3 \lambda) p_{0} e \Pi Y-\lambda \rho_{0} e \Delta Y\right], \\
& \quad \dot{k}_{0}, \\
k_{1}^{\prime}- & k_{0}^{\prime \prime}+\frac{2}{r} \dot{k}_{1}-2 H k_{1}^{\prime}+\frac{4}{r} k_{1} H-4 k_{0} \dot{H} \\
& -2 H^{2} k_{0}+\frac{k_{0}}{r^{2}} l(l+1) \\
= & -2 a^{3}(8 \pi+2 \lambda) u\left(\rho_{0}+p_{0}\right) \\
+ & {\left[(8 \pi+3 \lambda) p_{0}-\lambda \rho_{0}\right] 2 a^{2} k_{0} } \\
+ & 2 a^{2} e(8 \pi+4 \lambda) k_{0} p_{0} \Pi Y-2 a^{2} e \lambda k_{0} \rho_{0} \Delta, \\
\ddot{k_{1}}- & \dot{k}_{0}^{\prime}+\frac{2}{r} \dot{k}_{0}-\frac{2}{r^{2}} k_{1}-2 H \dot{k}_{1}-6 \dot{H} k_{1} \\
- & 2 H^{2} k_{1}+\frac{k_{1}}{r^{2}} l(l+1) \\
= & 2 a^{2} k_{1} p_{0}(8 \pi+2 \lambda)+2 \lambda a^{2} k_{1}\left(-\rho_{0}+p_{0}\right) \\
- & 2 a^{2} e \lambda k_{1} \rho_{0} \Delta \\
+ & 2 a^{2} e(8 \pi+4 \lambda) k_{1} p_{0} \Pi Y,
\end{aligned}
$$

where prime indicates the derivative with respect to $r$ and also, we have used the relation [35]

$\partial_{\theta} \partial_{\theta} Y=-l(l+1) Y-\cot \theta \partial_{\theta} Y$.

\section{Effects of axial gravitational waves}

In this section, we find expressions for the perturbation parameters $k_{0}, k_{1}, \Delta, \Pi, w, v$ and $u$. Equation (23) implies that either the factor $(8 \pi+2 \lambda)=0$, i.e., $\lambda=-4 \pi$ or $w\left(\rho_{0}+p_{0}\right)=0$. However, the viability conditions for $f(R, T)$ gravity models are

$f_{R}>0, \quad 1+\frac{f_{T}}{8 \pi}>0$ and $f_{R R}>0$,

and give the constraint $\lambda>-4 \pi$ for our model implying that $(8 \pi+2 \lambda) \neq 0$. Hence Eqs. (23) and (24) yield that $w=0$ and $v=0$. Substituting the unperturbed field equations in perturbed one, we obtain the following equations from (22), (25), (27) and (28), respectively.

$$
\begin{aligned}
& (8 \pi+3 \lambda) \rho_{0} \Delta-\lambda p_{0} \Pi=0, \\
& (8 \pi+3 \lambda) p_{0} \Pi-\lambda \rho_{0} \Delta=0, \\
& \dot{k}_{1}^{\prime}-k_{0}^{\prime \prime}+\frac{2}{r} \dot{k}_{1}-2 H k_{1}^{\prime}+\frac{4}{r} k_{1} H+\frac{k_{0}}{r^{2}} l(l+1) \\
& \quad=-2 a^{3}(8 \pi+2 \lambda) u\left(\rho_{0}+p_{0}\right) \\
& \quad+2 a^{2} e\left[(8 \pi+4 \lambda) k_{0} p_{0} \Pi Y-\lambda k_{0} \rho_{0} \Delta\right] \\
& \ddot{k}_{1}-\dot{k}_{0}^{\prime}+\frac{2}{r} \dot{k}_{0}-\frac{2}{r^{2}} k_{1}-2 H \dot{k}_{1}-2 \dot{H} k_{1}+\frac{k_{1}}{r^{2}} l(l+1) \\
& =2 a^{2} e\left[-\lambda k_{1} \rho_{0} \Delta+(8 \pi+4 \lambda) k_{1} p_{0} \Pi Y\right] .
\end{aligned}
$$

Solving (29) and (30) simultaneously for $\Pi$, we obtain

$\left((8 \pi+3 \lambda)^{2}-\lambda^{2}\right) p_{0} \Pi=0$,

which implies either

$\left((8 \pi+3 \lambda)^{2}-\lambda^{2}\right)=0$ or $\Pi=0$.

The first factor in the above equation yields $\lambda=-4 \pi$ and $-2 \pi$. However, keeping in mind the viability conditions for the assumed model, we exclude $\lambda=-4 \pi$. Hence if $\lambda=-2 \pi$, then there is a possibility that $\Pi \neq 0$ and similarly $\Delta \neq 0$, i.e., the axial GWs can affect the background matter in curvature-matter coupling scenario. Assuming the EoS for radiation dominated era $p_{0}=\frac{1}{3} \rho_{0}$, we obtain the following relationship between $\Pi$ and $\Delta$

$\Pi=3\left(\frac{8 \pi}{\lambda}+3\right) \Delta$.

Substituting the above relation in Eqs. (31) and (32), we are left with four unknowns $k_{0}, k_{1}, \Delta, u$ with three equations (26), (31), (32). Thus in order to have the system closed, we assume that GWs do not perturb the matter field, i.e., 
$\Delta=0=\Pi$. Now introducing a new quantity $Q(\eta, r)$ such that

$k_{1}(\eta, r)=r a(\eta) Q(\eta, r)$.

Using this equation with Eq. (26) in (32), we obtain

$$
\begin{aligned}
& \ddot{Q}-Q^{\prime \prime}+\frac{l(l+1)}{r^{2}} Q-a^{2} \\
& \quad\left[\frac{(4 \pi+3 \lambda)}{3} \rho_{0}-\frac{(12 \pi+5 \lambda)}{3} p_{0}\right] Q=0 .
\end{aligned}
$$

Inserting $p_{0}=\frac{\rho_{0}}{3}$, the values of $a(\eta)$ as well as $\rho_{0}$ from Eqs. (10) and (11) into (37), it follows that

$\ddot{Q}-Q^{\prime \prime}+\left[\frac{l(l+1)}{r^{2}}-\frac{4 c_{1}^{2} c_{2} b_{1} \lambda}{9} \eta^{\frac{6 \pi+2 \lambda}{6 \pi+\lambda} \frac{(-3)(8 \pi+\lambda)}{6 \pi+\lambda}}\right] Q=0$,

where $b_{1}=c_{1}^{\frac{-3(8 \pi+\lambda)}{6 \pi+\lambda}}$. Let us define $A=\frac{4 c_{1}^{2} c_{2} b_{1} \lambda}{9}$ and take $l=2$ such that the above equation becomes

$$
\ddot{Q}-Q^{\prime \prime}+\left[\frac{6}{r^{2}}-A \eta^{\frac{6 \pi+2 \lambda}{6 \pi+\lambda} \frac{(-3)(8 \pi+\lambda)}{6 \pi+\lambda}}\right] Q=0 .
$$

This is a wave equation and can be solved through separation of variables by assuming $Q(\eta, r)=\mathcal{T}(\eta) \mathcal{R}(r)$ and the initial conditions.

$Q(0, r)=\Psi_{1}(r), \quad \partial_{\eta} Q(0, r)=\Psi_{2}(r)$,

Introducing the separation constant $-m^{2}$, we obtain the following two differential equations

$$
\begin{aligned}
& \ddot{\mathcal{T}}-\left(A \eta^{\frac{6 \pi+2 \lambda}{6 \pi+\lambda} \frac{(-3)(8 \pi+\lambda)}{6 \pi+\lambda}}-m^{2}\right) \mathcal{T}=0, \\
& \mathcal{R}^{\prime \prime}-\left(\frac{6}{r^{2}}-m^{2}\right) \mathcal{R}=0 .
\end{aligned}
$$

These are second order homogeneous linear differential equations with variable coefficients. Equation (40) can yield some solution if the power of $\eta$ is fixed. So, we consider $\frac{6 \pi+2 \lambda}{6 \pi+\lambda} \frac{(-3)(8 \pi+\lambda)}{6 \pi+\lambda}=n$ and check that for what values of $n$, the values of $\lambda$ are consistent with viability criteria. We find that the values of $\lambda$ for $n>1$ are not consistent with $\lambda>-4 \pi$ (the viability criteria) and $n<-2$ yields imaginary values of $\lambda$. Hence, $n$ can have the values within the limit $-2 \leq n<1$. For $n=-2$, we have $\lambda=0$ which is the case of GR. For convenience, we consider the integer values in this interval, i.e., $n=0,-1$, to find the solution of Eq. (40). For $n=0$, the solution is

$\mathcal{T}(\eta)=c_{3} \cos m \eta+c_{4} \sin m \eta$, where $c_{3}$ and $c_{4}$ are constants of integration and for $n=-1$, we have

$$
\begin{aligned}
\mathcal{T}(\eta)= & c_{5} \eta e^{-i m \eta} \text { Hypergeometric } 1 \mathrm{~F} 1\left[1+\frac{A}{2 i m}, 2,2 i m \eta\right] \\
& +c_{6} \eta e^{-i m \eta} \text { Hypergeometric } \mathrm{U}\left[1+\frac{A}{2 i m}, 2,2 i m \eta\right],
\end{aligned}
$$

where $c_{5}, c_{6}$ are constants and Hypergeometric1F1, HypergeometricU are the confluent hypergeometric functions of the first and second kind, respectively. These functions are defined by

Hypergeometric1F1 $(\alpha ; \beta ; z)=\frac{\Gamma(\beta)}{\Gamma(\beta-\alpha) \Gamma(\alpha)} \int_{0}^{1} e^{z t} t$

$$
(\alpha-1)(1-t)^{\beta-\alpha-1} d t
$$

Hypergeometric $\mathrm{U}(\alpha, \beta, z)=\frac{1}{\Gamma(\alpha)} \int_{0}^{\infty} e^{-z t} t(\alpha-1)$

$$
(1+t)^{\beta-\alpha-1} d t
$$

where " $\Gamma$ " indicates the gamma function. The solution of Eq. (41) is obtained as

$$
\begin{aligned}
\mathcal{R}(r)= & \sqrt{\frac{2}{m \pi}} c_{7}\left(\frac{-3 \cos m r}{m r}-\sin m r+\frac{3 \sin m r}{m^{2} r^{2}}\right) \\
& +\sqrt{\frac{2}{m \pi}} c_{8}\left(\frac{-3 \cos m r}{m^{2} r^{2}}-\frac{3 \sin m r}{m r}+\cos m r\right),
\end{aligned}
$$

where $c_{7}, c_{8}$ are integration constants. Inserting the values of $\mathcal{R}(r)$ and $\mathcal{T}(\eta)$ in $Q(\eta, r)=\mathcal{T}(\eta) \mathcal{R}(r)$, we obtain $Q(\eta, r)$ for both values of $n$. Furthermore, using initial conditions one can find the expressions for $\Psi_{1}(r)$ and $\Psi_{2}(r)$ for $n=0$ as well as $n=-1$.

Replacing the values of $Q(\eta, r)$ and $a(\eta)$ in Eq. (36), we obtain the value of $k_{1}$ while the expression for $k_{0}$ is obtained from Eq. (26) as follows

$k_{0}=B(r)+\int_{\eta_{0}}^{\eta} k_{1}^{\prime}(\tau, r) d \tau$,

where $\eta_{0}$ is the conformal time at the hypersurface originating GWs. Assuming $k_{0}(\eta, r)=0$, we have $B(r)=0$ and $k_{0}$ becomes

$k_{0}=(r \mathcal{R}(r))^{\prime} \int_{\eta_{0}}^{\eta} a(\tau) \mathcal{T}(\tau) d \tau$.

Finally, replacing the values of $k_{0}, k_{1}$ and $\Delta=0=\Pi$ in Eq. (31), we obtain for $n=0$

$$
\begin{aligned}
u(\eta, r)= & \frac{\eta^{\frac{\lambda}{6 \pi+\lambda}} c_{1}}{(m r)^{\frac{5}{2}}} \sqrt{\frac{2 r}{\pi}}\left[\left(3 m r c_{7}+m^{3} r^{3} c_{7}+3 c_{8}\right) \cos m r\right. \\
& \left.+\left(-3 c_{7}+m r\left(3+m^{2} r^{2}\right) c_{8}\right) \sin m r\right] \\
& {\left[\left(c_{3}-m \eta c_{4}\right) \cos m \eta+\left(m \eta c_{3}+c_{4}\right) \sin m \eta\right] . }
\end{aligned}
$$


For $n=-1$, we have

$$
\begin{aligned}
u(\eta, r)= & \frac{\eta^{\frac{6 \pi+2 \lambda}{6 \pi+\lambda}} e^{-i m \eta} c_{1}}{(m r)^{\frac{5}{2}}} \sqrt{\frac{r}{2 \pi}}\left[\left(1-\frac{6 \pi+2 \lambda}{6 \pi+\lambda}+i m \eta\right)\right. \\
& \times\left\{2 c_{5} \text { Hypergeometric1F1 }\left[1+\frac{A}{2 i m}, 2,2 i m \eta\right]\right. \\
& \left.+2 c_{6} \text { Hypergeometric U }\left[1+\frac{A}{2 i m}, 2,2 i m \eta\right]\right\} \\
& \left.+(A+2 i m) \eta\left\{2 c_{6} \text { HypergeometricU }\right]\right\} \\
& \times\left[2+\frac{A}{2 i m}, 3,2 i m \eta\right]-c_{5} \\
& \left.\left.\times \text { Hypergeometric1F1 }\left[2+\frac{A}{2 i m}, 3,2 i m \eta\right]\right\}\right] \\
& \times\left[\left(3 m r c_{7}+m^{3} r^{3} c_{7}+3 c_{8}\right) \cos m r\right. \\
& \left.+\left(-3 c_{7}+m r\left(3+m^{2} r^{2}\right) c_{8}\right) \sin m r\right] .
\end{aligned}
$$

Thus the final expression for four velocity in radiation dominated phase becomes

$V_{\alpha}=\left(-c_{1} \eta^{\frac{6 \pi+2 \lambda}{6 \pi+\lambda}}, 0,0, e \partial_{\theta} Y u(\eta, r) \sin \theta\right)$.

Also, $Y=Y_{20}(\theta)=\frac{1}{4} \sqrt{\frac{5}{\pi}}\left(3 \cos ^{2} \theta-1\right)$ leads to $\partial_{\theta} Y=$ $\frac{1}{4} \sqrt{\frac{5}{\pi}} \cos \theta \sin \theta$ and hence

$V_{\alpha}=\left(-c_{1} \eta^{\frac{6 \pi+2 \lambda}{6 \pi+\lambda}}, 0,0, \frac{e}{4} \sqrt{\frac{5}{\pi}} u(\eta, r) \cos \theta \sin ^{2} \theta\right)$.

Thus the azimuthal velocity of any point $\mathrm{P}$ having coordinates $(\eta, r, \theta, \phi)$ is $V_{3}=\frac{e}{4} \sqrt{\frac{5}{\pi}} u(\eta, r) \cos \theta \sin ^{2} \theta$, where $u(\eta, r)$ is given in Eqs. (46) and (47) for $n=0,-1$, respectively.

\section{Final remarks}

According to rough approximate, a pair of massive black holes merge in every $223_{-115}^{352} \mathrm{sec}$ and a binary of neutron star merge in every $13_{-9}^{49} \sec$ [43]. Among these mergers a small fraction is detected by advance interferometers of LIGOVirgo collaboration and can be associated to some individual GW event. The rest of the events contribute to make a stochastic background which is a random GW signal originated by various independent, weak and unresolved sources. These sources include for instance, the supernova explosions at the end of a massive star's life (including non-symmetric explosions), a rapidly rotating neutron star, cosmic strings etc. Mathematical and statistical approaches have been developed to observe these stochastic background of GWs and extract information from them $[44,45]$. These GWs signals have great influence on cosmic evolution and hence the study of different aspect of GW phenomenon is very significant.

The main goal of this manuscript is to explore the changes produced by axial GWs in geometry as well as matter of a flat universe during evolution and in the context of curvaturematter coupling theory. For this purpose, we assume the presence of these waves and find the corresponding geometrical and material changes produced by these waves in $f(R, T)$ gravity. We have introduced axial perturbations in the flat FRW spacetime, the background matter is also perturbed as well as the four velocity is allowed to be non-comoving. We then proceed to find all unknown parameters of perturbations with the help of perturbed and unperturbed field equations. It is mentioned here that all field equations reduce to GR equations [36] for $\lambda=0$.

The factors $w, v$, appearing in $V_{1}$ and $V_{2}$ are zero showing that axial waves do not change these components of velocity which is similar to that in GR. We have found that axial GWs in $f(R, T)$ theory can perturb the background matter in contrast to GR. However, here we suppose $\Delta$ and $\Pi$ equal to zero in order to find the remaining functions $k_{0}, k_{1}$ and $u$. The resulting $k_{0}$ and $k_{1}$ are different from those of GR and depend upon the coupling constant $\lambda$. The function $u$ appearing in the azimuthal velocity component has non-zero expression showing that fluid exhibits a rotation due to axial GWs similar to GR. But the expression of $u$ here depends upon $\lambda$ and differs from GR.

Currently, our universe is in expansion phase and it is crucial to investigate the propagation of GWs in this expanding universe. In this regard, we expand our analysis using the EoS $p_{0}=-\rho_{0}$ for expanding matter and observe how such types of GWs can perturb the flat cosmos in the recent era. For $p_{0}=-\rho_{0}$, the scale factor and density have the expressions

$a(\eta)=\frac{\tilde{c}_{1}}{\eta}, \quad \rho_{0}=\tilde{c}_{2} a^{\frac{-3 \lambda}{2 \pi}}$,

where $\tilde{c}_{1}$ and $\tilde{c}_{2}$ are integration constants. It is found that this EoS can yield non-vanishing $w$ and $v$ (from (23) and (24)) while the remaining expressions remain the same with $A=\frac{8(2 \pi+\lambda)}{3} c_{1}^{2} c_{2} b_{1}, b_{1}=\tilde{c}_{6}^{\frac{-3 \lambda}{2 \pi}}$ and $\frac{3 \lambda-4 \pi}{2 \pi}=n$. In dark energy dominated phase, $n$ can take positive and negative values, however, similar to radiation dominated phase, $n=$ -2 yields the GR case.

The angular $(\Omega)$ and linear rotational $(V)$ velocities of the fluid are

$\Omega=\frac{V^{3}}{V^{0}}=\frac{C u}{a r^{2}} \cos \theta ; \quad C=\frac{e}{4} \sqrt{\frac{5}{\pi}}$,

$V=\operatorname{ar} \sin \theta \Omega=\frac{C u \sin 2 \theta}{2 r}$.

When the expression of $u(\eta, r)$ is continuous at the wave front, the smooth wave profile does not induce any cosmological rotation [36]. Hence we conclude that the axial GW can induce a cosmological rotation if $u(\eta, r)$ is discontinuous at the wave front. If the freely falling particles are displaced by a GW, it is called memory effect of the GW. Hence the 
axial GW in $f(R, T)$ gravity induces memory effect when the wave profile has discontinuity at the wave front. Also, the model considered here describes the simplest curvaturematter coupling and we assume this model to reduce the calculation work. However, this work can be extended for other minimally coupled models containing nonlinear power of $R$ or $T$ or non-minimally coupled models leading to interesting results. Such models may yield the non-vanishing values of the perturbation parameters which are zero in the present scenario.

When a GW without memory passes through a detector, it produces an oscillatory deformation and returns the detector back to its equilibrium state. On the other hand, a GW with memory can induce a permanent deformation in an idealized detector, i.e., a truly free falling detector [46]. The detectors like Weber bars and LIGO are not sensitive to the memory effect. However, the detectors of the type like LISA (Laser interferometry space antenna) or advanced LIGO can detect the memory due to its sensitivity and with strong memory sources $[47,48]$. Also, the ground-based detectors are not truly free falling and cannot store a memory signal while LISA like detectors are able to maintain the permanent displacement because these are free floating.

Acknowledgements We would like to thank the Higher Education Commission, Islamabad, Pakistan for its financial support through the Indigenous Ph.D. 5000 Fellowship Program Phase-II, Batch-III.

Open Access This article is distributed under the terms of the Creative Commons Attribution 4.0 International License (http://creativecomm ons.org/licenses/by/4.0/), which permits unrestricted use, distribution, and reproduction in any medium, provided you give appropriate credit to the original author(s) and the source, provide a link to the Creative Commons license, and indicate if changes were made. Funded by SCOAP ${ }^{3}$.

\section{References}

1. H.A. Buchdahl, Mon. Not. R. Astron. Soc. 150, 1 (1970)

2. S. Nojiri, S.D. Odintsov, Phys. Lett. B 631, 1 (2005)

3. T. Harko, F.S.N. Lobo, S. Nojiri, S.D. Odintsov, Phys. Rev. D 84, 024020 (2011)

4. P. Ratra, L. Peebles, Phys. Rev. D 37, 3406 (1988)

5. R.R. Caldwell, R. Dave, P.J. Steinhardt, Phys. Rev. Lett. 80, 1582 (1998)

6. R.R. Caldwell, Phys. Lett. B 545, 23 (2002)
7. N. Afshordi, D.J.H. Chung, G. Geshnizjani, Phys. Rev. D 75, 083513 (2007)

8. M.R. Setare, Phys. Lett. B 648, 329 (2007)

9. M.R. Setare, Phys. Lett. B 653, 116 (2007)

10. S. Chaplygin, Sci. Mem. Mosc. Univ. Math. Phys. 21, 1 (1904)

11. AYu. Kamenshchik, U. Moschella, V. Pasquier, Phys. Lett. B 511, $265(2001)$

12. M.C. Bento, O. Bertolami, A.A. Sen, Phys. Rev. D 66, 043507 (2002)

13. A. De Felice, S. Tsujikawa, Living Rev. Relativ. 13, 3 (2010)

14. S.I. Nojiri, S.D. Odintsov, Phys. Rep. 505, 59 (2011)

15. T. Harko, F.S.N. Lobo, Galaxies 2, 410 (2014)

16. M. Sharif, M. Zubair, J. Cosmol. Astropart. Phys. 03, 28 (2012)

17. H. Shabani, M. Farhoudi, Phys. Rev. D 90, 044031 (2014)

18. Z. Yousaf, M.Z. Bhatti, Ume Farwa, Class. Quantum Gravity 34, 145002 (2017)

19. M. Sharif, A. Siddiqa, Eur. Phys. J. Plus 132, 529 (2017)

20. M. Sharif, A. Siddiqa, Int. J. Mod. Phys. D 27, 1850065 (2018)

21. B.P. Abbott et al., Phys. Rev. Lett. 116, 061102 (2016)

22. B.P. Abbott et al., Phys. Rev. Lett. 118, 221101 (2017)

23. B.P. Abbott et al., Phys. Rev. Lett. 119, 161101 (2017)

24. H.R. Kausar, L. Philippoz, P. Jetzer, Phys. Rev. D 93, 124071 (2016)

25. M.E.S. Alves, P.H.R.S. Moraes, J.C.N. de Araujo, M. Malheiro, Phys. Rev. D 94, 024032 (2016)

26. M. Sharif, A. Siddiqa, Phys. Dark Universe 15, 105 (2017)

27. M. Sharif, A. Siddiqa, Astrophys. Space Sci. 362, 226 (2017)

28. T. Regge, J.A. Wheeler, Phys. Rev. 108, 1063 (1957)

29. F.J. Zerilli, Phys. Rev. D 02, 2141 (1970)

30. S.W. Hawking, Phys. Rev. Lett. 26, 1344 (1971)

31. R.V. Wagoner, Astrophys. J. 278, 345 (1984)

32. E. Malec, G. Wylężek, Class. Quantum Gravity 22, 3549 (2005)

33. O. Svitek, J. Phys. Conf. Ser. 229, 012070 (2010)

34. S. Viaggiu, Class. Quantum Gravity 34, 035018 (2017)

35. W. Kulczycki, E. Malec, Class. Quantum Gravity 34, 135014 (2017)

36. W. Kulczycki, E. Malec, Phys. Rev. D 96, 063523 (2017)

37. U.H. Gerlach, U.K. Sengupta, Phys. Rev. D 19, 2268 (1979)

38. U.H. Gerlach, U.K. Sengupta, Phys. Rev. D 22, 1300 (1980)

39. C. Gundlach, J.M. Martin-Garcia, Phys. Rev. D 61(2000), 084024 (2000)

40. C. Clarkson, T. Clifton, S. February, J. Cosmol. Astropart. Phys. 06, 25 (2009)

41. N.J. Poplawski, arXiv:gr-qc/0608031

42. V. Faraoni, Phys. Rev. D 80, 124040 (2009)

43. B.P. Abbott et al., Phys. Rev. Lett. 120, 091101 (2018)

44. B. Allen, J.D. Romano, Phys. Rev. D 59, 102001 (1999)

45. R. Smith, E. Thrane, Phys. Rev. X 8, 021019 (2018)

46. M. Favata, Class. Quantum Gravity 27, 084036 (2010)

47. P.D. Lasky, E. Thrane, Y. Levin, J. Blackman, Y. Chen, Phys. Rev. Lett. 117, 061102 (2016)

48. P.M. Zhang, C. Duval, G.W. Gibbons, P.A. Horvathy, Phys. Lett. B 772, 743 (2017) 